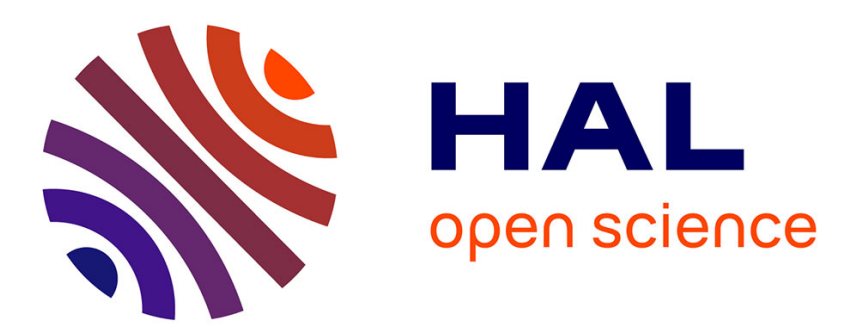

\title{
Wave celerity on a shear-thinning fluid film flowing down an incline
}

Séverine Millet, Valéry Botton, François Rousset, Hamda Ben Hadid

\section{To cite this version:}

Séverine Millet, Valéry Botton, François Rousset, Hamda Ben Hadid. Wave celerity on a shear-thinning fluid film flowing down an incline. Physics of Fluids, 2008, 20 (3), pp.031701. 10.1063/1.2889140 . hal-00444988

\section{HAL Id: hal-00444988 \\ https://hal.science/hal-00444988}

Submitted on 14 Jun 2012

HAL is a multi-disciplinary open access archive for the deposit and dissemination of scientific research documents, whether they are published or not. The documents may come from teaching and research institutions in France or abroad, or from public or private research centers.
L'archive ouverte pluridisciplinaire HAL, est destinée au dépôt et à la diffusion de documents scientifiques de niveau recherche, publiés ou non, émanant des établissements d'enseignement et de recherche français ou étrangers, des laboratoires publics ou privés. 


\title{
Wave celerity on a shear-thinning fluid film flowing down an incline
}

\author{
S. Millet, ${ }^{1}$ V. Botton, ${ }^{1}$ F. Rousset, ${ }^{2}$ and H. Ben Hadid ${ }^{1}$ \\ ${ }^{1}$ Université de Lyon/Université Lyon 1/INSA-Lyon/ECL, \\ Laboratoire de Mécanique des Fluides et d'Acoustique, UMR CNRS 5509, Bât. J. Jacquard, \\ 20 av. A. EINSTEIN, 69621 Villeurbanne Cedex, France \\ ${ }^{2}$ CETHIL/UMR CNRS 5008, Université de Lyon/INSA-Lyon/Université Lyon 1, Bat. Sadi Carnot, 9, \\ Rue de la Physique, 69621 Villeurbanne Cedex, France
}

(Received 9 November 2007; accepted 4 February 2008; published online 27 March 2008)

This letter presents a phenomenological model predicting the celerity of long surface waves on a non-Newtonian fluid flowing down an inclined plane. We show that, for a shear-thinning fluid, the celerity is greater than the well-known value $c=2 U_{0}$. The developed model points at the significant effect of the viscosity disturbance and also provides a likely explanation for the decrease in threshold for the instability. (c) 2008 American Institute of Physics. [DOI: 10.1063/1.2889140]

The stability of a Newtonian viscous film flow down an incline was addressed by many authors, among them Yih $^{1}$ and Benjamin. ${ }^{2}$ The main features brought about by these authors are that the instability occurs at very long waves compared to the thickness of the film, and inertia plays a major role in the destabilization of the flow. The long surface waves travel with a celerity $c$ of twice the free surface speed $U_{0}$ of the flow and the value of the threshold is given by $\operatorname{Re}_{c}=(5 / 6) \cot \beta$, where $\beta$ is the angle of inclination and $\operatorname{Re}_{c}$ is the Reynolds number based on the average velocity.

In particular, $\mathrm{Smith}^{3}$ proposed a complete scenario based on a long wave expansion of the linearized problem. His approach not only shows very clearly the mechanisms of the instability but it also gives a simple way to compute the former values of $\mathrm{Re}_{c}$ and celerity. He notably puts to light that the main inertia term responsible for the destabilization is that of advection by the base flow in a framework travelling with waves. This term is thus proportional to $(U-c), U$ being the base flow velocity, so that the instability mechanism is directly linked to wave celerity.

However, in many cases, in industrial (coating processes) or environmental configurations (muds, debris or lava flows), the fluid is not Newtonian but more often shearthinning, i.e., its viscosity locally decreases with increasing shear rates. The stability of a non-Newtonian film flowing down an incline has thus been the object of several systematic studies for different chosen constitutive laws (see Rousset et $a l^{4}$ for a brief review). In particular, Rousset et al., ${ }^{4}$ have shown in the case of a shear-thinning Carreau fluid that the waves travel faster than in that of a Newtonian one and also that the instability is triggered at a lower Reynolds number. These authors also pointed at the fact that the decrease in $\operatorname{Re}_{c}$ could be a consequence of the increased difference $(U-c)$. Moreover, a recent study by Nouar et $a .^{5}$ has shown that the viscosity disturbance due to the perturbation can affect the stability of shear-thinning Poiseuille flows.

To our knowledge, no general phenomenological approach has yet been conducted to explain these features. We propose here to walk in Smith's footprints towards a deeper understanding of why shear-thinning means faster waves and a lower instability threshold. Note that a similar conclusion has recently been drawn by Nouar et $a l^{5}$ for a Poiseuille flow. For doing so, we evaluate the relative contribution of shear-thinning arising from the base flow on the one hand and from the perturbation flow on the other hand.

Let us extend Smith's approach to the case of a purely viscous film flowing down an incline, as presented in Fig. 1. Note that the equations of the problem are made dimensionless with the same characteristic parameters as those adopted in Rousset et $a l^{4}$ The established base flow $U$ occurs under the balance of shear force and the weight longitudinal component which is written in dimensionless form as

$$
\eta \frac{\partial U}{\partial y}=-y \text {. }
$$

The viscosity $\eta$ is assumed here to be a function of the shear rate $\dot{\gamma}=-\partial U / \partial y$ only, with $\eta=1$ at zero shear. We assume a small deformation of the free surface of amplitude $\xi$; it is sinusoidal with a far greater wavelength than the thickness of the film. This deformation results in a shear stress, $\tau_{\mathrm{i}}$, at the free surface which can be evaluated through the following expansion:

$$
\tau_{i}=\left.\eta \frac{\partial U}{\partial y}\right|_{\xi}=\eta_{0}\left(\left.\frac{\partial U}{\partial y}\right|_{0}+\left.\xi \frac{\partial^{2} U}{\partial y^{2}}\right|_{0}\right)=\left.\xi \frac{\partial^{2} U}{\partial y^{2}}\right|_{0} .
$$

Note that we have neglected here the change in viscosity at the deformed free surface under the effect of this small shear stress, so that $\eta(\xi)=\eta_{0}=1$. Combining Eq. (2), the derivative of Eq. (1), and the no shear condition at the free surface, one gets

$$
\tau_{i}=-\xi \quad \text { at } y=0 .
$$

The perturbation flow offsets this shear stress such that there is no shear at the deformed interface. The shear stress perturbation $\tau_{i}^{\prime}$ at the free surface is thus

$$
\tau_{i}^{\prime}=\xi \quad \text { at } y=0 .
$$

This perturbation shear stress is in phase with the free surface deformation and generates a secondary flow which is in the same direction as the main flow under the crests, in the 


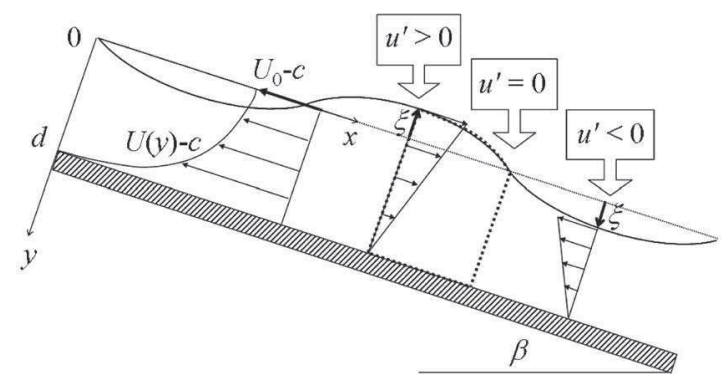

FIG. 1. Flow configuration, control volume, and perturbation profiles in the framework of the wave for a Newtonian fluid.

opposite direction under the lows and zero under the nodes (see Fig. 1). This perturbation flow is parallel under the long wave assumption and at the leading order in wave number $\alpha$. In the Newtonian case, the amplitude of the velocity perturbation $u^{\prime}$, thus has a linear Couette-type profile. In the shearthinning case, as the shear rate increases with the depth $y$, the viscosity decreases. The profile then has a curvature which increases with $y$. This is illustrated in Fig. 2 showing perturbation profiles for Newtonian and shear-thinning fluids (see Rousset et $a l^{4}$ for details on the numerical computation).

At first order in $\alpha, \mathrm{Smith}^{3}$ has shown that, for Newtonian fluids, the flow is neutrally stable since inertia is necessary to trigger the instability and is not accounted for at this order. This still holds for the case of shear-thinning fluids. However, the leading order approach allows us to evaluate the celerity of the surface waves in the neutral case. One relies on this for a mass budget in the framework of the wave. Let us consider the control volume in dashed lines in Fig. 1; in this frame, the base flow profile is written as $[U(y)-c]$. The mass budget thus gives

$$
\int_{-\xi}^{d}[U(y)-c] d y+\int_{-\xi}^{d} u^{\prime} d y=\int_{0}^{d}[U(y)-c] d y .
$$

The LHS represents the inflow at the left side of the control volume. These terms represent the base flow and the leading order perturbation flow contributions. The term in the RHS is denoted as the outflow through the right side of control volume, where the longitudinal perturbation velocity is zero. Linearizing Eq. (5) yields

$$
\xi\left[U_{0}-c\right]=q^{\prime} \quad \text { with } q^{\prime}=\int_{0}^{d} u^{\prime} d y,
$$

so that the celerity is directly bonded to the perturbation flow rate $q$ '. The question is thus: "How does the rheology affect the perturbation flow-rate $q^{\prime}$ (for a given base flow rate)?", or with respect to Fig. 2, "Is the surface delimited by the curved profile always greater than that delimited by the linear dashed profile?"

The integral in Eq. (6) is evaluated by parts so that the celerity is written as a function of the perturbation shear-rate,

$$
c=U_{0}+\frac{1}{\xi} \int_{0}^{d} y \frac{\partial u^{\prime}}{\partial y} d y .
$$

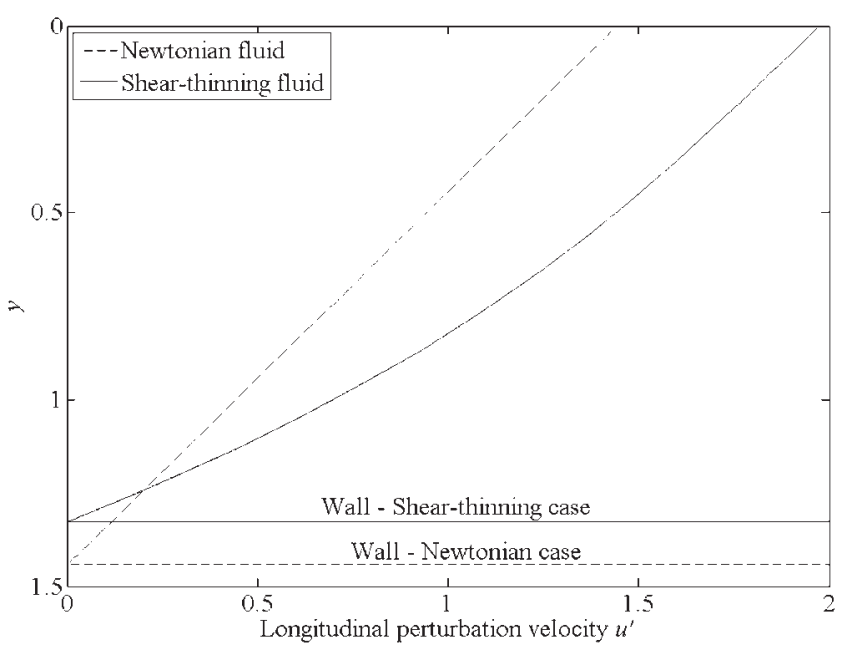

FIG. 2. Longitudinal perturbation profiles $u^{\prime}$ under a crest for a Newtonian fluid $(L=0)$ and a shear-thinning fluid $(L=1)$ in the neutral case with $\beta=0.2 \mathrm{rad}, I=10^{-3}$, and $n=0.5$.

Isolating the contribution of shear-thinning arising from the base flow from that due to the perturbation flow, one can write

$$
\eta(y) \frac{\partial u^{\prime}}{\partial y}+\eta^{\prime}(y) \frac{\partial U}{\partial y}=\tau_{i}^{\prime}
$$

where terms proportional to $\partial v^{\prime} / \partial x$ have been neglected in front of $\partial u^{\prime} / \partial y$. Here, the viscosity profile $\eta(y)$ is solely prescribed by the base flow through the constitutive law of the fluid. The second term is due to the viscosity perturbation $\eta^{\prime}(y)$ under the influence of additional stresses, themselves yielded by the perturbation flow. This viscosity perturbation can be negative or positive whether the additional shear has the same sign as the base flow shear-stress or the opposite sign. Here, for a shear-thinning fluid, it is negative under the crests, and positive under the lows. This viscosity perturbation can be evaluated by considering small variations, $d \dot{\gamma}$, of the shear-rate around its base value, $\dot{\gamma}$; one then writes $\eta^{\prime}=(\partial \eta / \partial \dot{\gamma}) d \dot{\gamma}$. Expanding and linearizing the strain rates tensor then yields $d \dot{\gamma}=\dot{\gamma}\left(\partial u^{\prime} / \partial y\right) /(\partial U / \partial y)$. In the present notations, $\dot{\gamma}=-\partial U / \partial y$, thus, $\eta^{\prime}=-(\partial \eta / \partial \dot{\gamma})\left(\partial u^{\prime} / \partial y\right)$ and relation (8) becomes

$$
\eta(y)\left(1+\frac{\dot{\gamma}}{\eta} \frac{\partial \eta}{\partial \dot{\gamma}}\right) \frac{\partial u^{\prime}}{\partial y}=\tau_{i}^{\prime} .
$$

One can first check that, in relation (9), the LHS corresponds to the expression of the shear stress given by Rousset et al.. ${ }^{4}$ Eventually, the perturbation flow rate is written as

$$
\begin{aligned}
q^{\prime} & =-\int_{0}^{d} \frac{\tau_{i}^{\prime} y}{\eta}\left(1+\frac{\dot{\gamma}}{\eta} \frac{\partial \eta}{\partial \dot{\gamma}}\right)^{-1} d y \\
& =-\int_{0}^{d} \frac{\tau_{i}^{\prime} y}{\eta} d y-\int_{0}^{d} \frac{\tau_{i}^{\prime} y}{\eta}\left(1+\frac{\dot{\gamma}}{\eta} \frac{\partial \eta}{\partial \dot{\gamma}}\right)^{-1} \cdot\left(-\frac{\dot{\gamma}}{\eta} \frac{\partial \eta}{\partial \dot{\gamma}}\right) d y .
\end{aligned}
$$




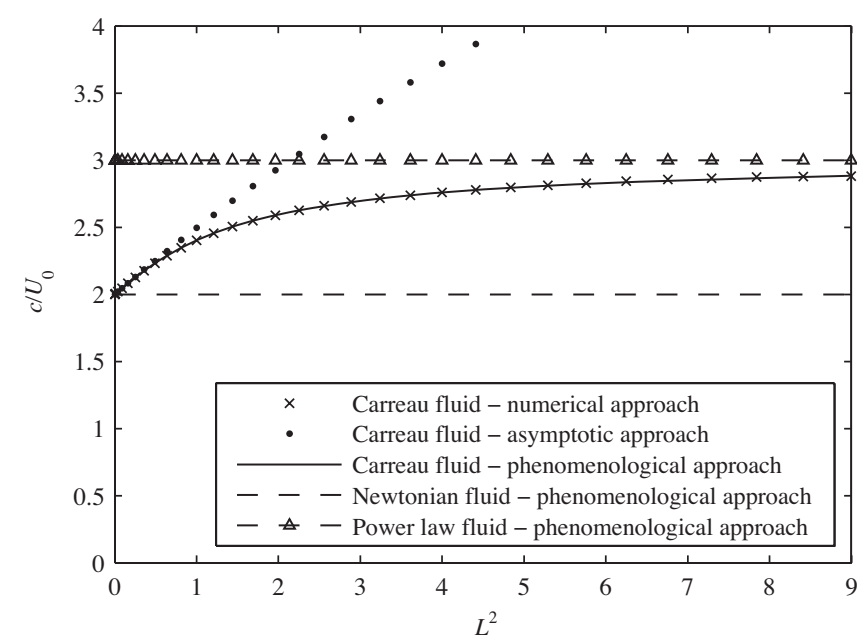

FIG. 3. Celerity in the neutral case over free surface velocity as a function of $L^{2}$ for $\beta=0.2 \mathrm{rad}, I=10^{-3}$, and $n=0.5$.

Combining Eqs. (1), (4), (7), and (10) provides us with an evaluation of the celerity as a function of the base flow and the constitutive law of the fluid,

$$
c=2 U_{0}-\int_{0}^{d} \dot{\gamma}\left[\frac{\frac{\dot{\gamma}}{\eta} \frac{\partial \eta}{\partial \dot{\gamma}}}{1+\frac{\dot{\gamma}}{\eta} \frac{\partial \eta}{\partial \dot{\gamma}}}\right] d y .
$$

A first estimate of the celerity can be found by neglecting the viscosity perturbation in Eq. (8). This leads back to Yih's famous statement, $c=2 U_{0}$. Let us recall ${ }^{4}$ here that, for a given flow rate, shear-thinning results in an increase in free-surface velocity $U_{0}$ so that the celerity can be expected to be greater in this case. A step further is made by accounting for the viscosity perturbation through the integral in Eq. (11). For any shear-thinning fluid, the integral is negative, so that the waves travel faster than twice the free surface velocity.

Considering the case of a shear-thinning fluid, modelled by the Carreau rheological law, the viscosity is written as

$$
\eta=I+(1-I)\left[1+(L \dot{\gamma})^{2}\right]^{(n-1) / 2},
$$

with $L$ a dimensionless constant of the material, $I$ the ratio of high shear rate over low shear rate Newtonian viscosities, and $n$ the power law exponent $(0<n<1$ for a shear-thinning fluid). The additional term within the brackets in Eq. (11), $(\dot{\gamma} / \eta)(\partial \eta / \partial \dot{\gamma})$, correspond to the slope of the constitutive law of the fluid in a log-log plot. In the case of a shearthinning Carreau fluid, the slope decreases from 0 at the free surface to a negative value greater than the slope of the power-law region, as $(n-1)$. The results of Eq. (11) with a Carreau constitutive law (12) are plotted as a full line in Fig. 3. As shown in the figure, in the whole range of the investigated $L$ values, these results are in good agreement with the numerical solutions (crosses) obtained by Rousset et al. ${ }^{4}$ from the resolution of the complete Orr-Sommerfeld equation. For $L \rightarrow 0$, they also agree well with the asymptotic analysis results (dots) of these authors under the assumptions of long waves and weakly non-Newtonian behavior. The

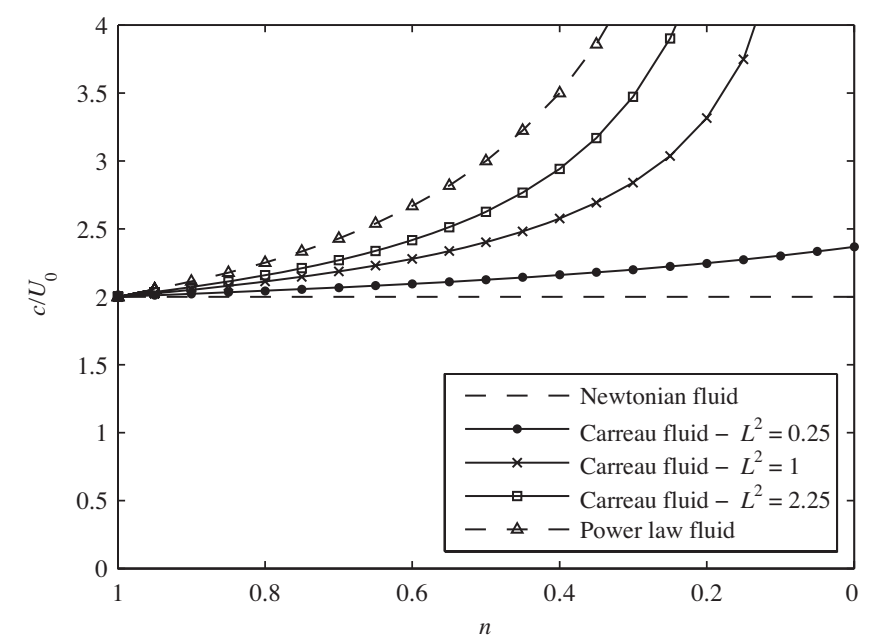

FIG. 4. Celerity in the neutral case over free surface velocity as a function of $n$ for $\beta=0.2 \mathrm{rad}, I=10^{-3}$, and $L^{2}=0,0.25,1,2.25$ and for a power law fluid.

base flow is computed numerically to evaluate the thickness $d$ and the shear-rate profile $\dot{\gamma}(y)$ that appears in the integrand, whereas $(\dot{\gamma} / \eta)(\partial \eta / \partial \dot{\gamma})$ is expressed analytically as a function of $\dot{\gamma}$.

For high shear rates and small values of parameter $I$, the Carreau model tends towards a power law model. The viscosity is expressed as

$$
\eta=L^{n-1} \dot{\gamma}^{n-1},
$$

and from Eq. (11), the celerity reduces to

$$
c=U_{0}\left(1+\frac{1}{n}\right) .
$$

This case is represented in Fig. 3 through a dashed line. One has to notice that, though the celerity is in order of magnitude twice the free surface velocity, the influence of the viscosity perturbation is not fully negligible.

Figure 4 represents the celerity over the free surface velocity as a function of $n$ obtained with the present approach for three constitutive laws: Newtonian, Carreau, and power laws. For the Carreau model, different values of $L$ are presented showing the separated influence of $L$ and $n$. From these results, it is clear that the viscosity perturbation is able to play a significant part in the instability mechanisms. In particular, the main destabilizing inertia effects appearing at higher order in $\alpha$ are proportional to $\left(U_{0}-c\right)$. They increase thus as the shear-thinning gets stronger and the flow is expected to be more unstable in the shear-thinning case than in the Newtonian case.

In this work, we present a phenomenological model predicting the long wave celerity in the neutral case of a nonNewtonian fluid flowing down an inclined plane. We extended Smith's model for Newtonian fluids to any purely viscous fluid. We show that, for a shear-thinning fluid, the celerity is still greater than the well known value $c=2 U_{0}$. The developed model points at the significant effect of the viscosity disturbance. The obtained results show a good agreement with those from Rousset et $a .^{4}$ in the case of a Carreau model. The model also provides a likely explanation 
for the decrease in threshold for the instability: the advection by the base flow in the framework of the wave, proportional to $\left(c-U_{0}\right)$ is stronger for a shear-thinning fluid.

${ }^{1}$ C.-S. Yih, "Stability of liquid flow down an inclined plane," Phys. Fluids 6, 321 (1963).

${ }^{2}$ T. B. Benjamin, "Wave formation in laminar flow down an inclined plane," J. Fluid Mech. 2, 554 (1957).
${ }^{3}$ M. K. Smith, "The mechanism for the long-wave instability in thin liquid films," J. Fluid Mech. 217, 469 (1990).

${ }^{4}$ F. Rousset, S. Millet, V. Botton, and H. BenHadid, "Temporal stability of Carreau fluid flow down an incline," J. Fluids Eng. 129, 913 (2007).

${ }^{5}$ C. Nouar, A. Bottaro, and J. P. Brancher, "Delaying transition to turbulence in channel flow: Revisiting the stability of shear thinning fluids," J. Fluid Mech. 592, 177 (2007). 